\title{
Synthesis of nitrogen-containing dispiroheterocycles (1) using nitrilimines
}

\author{
Hany M. Dalloul* \\ Chemistry Department, Faculty of Science, Al-Aqsa University of Gaza, \\ P.O.Box 4051, Gaza, Palestine \\ E-mail: hanydallool@yahoo.com
}

\begin{abstract}
A novel series of 1,2,4,5,10,11,13,14-octaazadispiro[5.2.5.2] hexadeca-2,12-dienes 4a-k were synthesized by the reaction of 1,4-cyclohexanedione methylhydrazone $\mathbf{3}$ with appropriate nitrilimines 2 . The microanalysis and the spectral data of the synthesized compounds are in full agreement with their molecular structure.
\end{abstract}

Keywords: Nitrilimines, 1,4-cyclohexanedione methylhydrazone, dispiroheterocycles

\section{Introduction}

In recent years, attention has been increasing regarding the synthesis of spiroheterocyclic compounds which exhibit various biological activities, ${ }^{1-8}$ pharmaceutical $^{9,10}$ and antitumor properties. $^{11,12}$ Nitrilimines are well explored dipoles and their reactions for the construction of heterocyclic systems are known to proceed via 1,3-dipolar cycloaddition with multiple bonds, cyclocondensation with nucleophilic substrates and 1,3-electrophilic addition with nucleophiles. Examples of these modes of reactions were recently reviewed by Ferwanah et al. ${ }^{13}$ for the reactions of hydrazones and oximes with nitrilimines and nitrile oxides. The synthesis of different spiroheterocycles I-III was recently reported using nitrilimines and cycloalkanone hydrazones or oximes $^{14-17}$ (Figure 1). As part of our program aimed at developing new biologically active compounds, we report here the synthesis of the hitherto unknown dispiroheterocyclic compounds 4a-k from the reaction between different nitrilimines $\mathbf{2}$ and 1,4-cyclohexanedione methyl hydrazone 3 . 


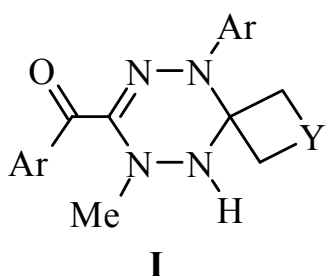

I<smiles>[Y17]C(=O)C1=NN([Y17])C2(C[Y]C2)N1</smiles>

II<smiles>[R]N1CCC2(CC1)N([Al])N=C(C(=O)[Al])N2[Y]</smiles>

III

$\mathrm{Ar}=\mathrm{Ph}-, 2-\mathrm{C}_{10} \mathrm{H}_{7^{-}}, 2-\mathrm{C}_{4} \mathrm{H}_{3} \mathrm{O}-$, 2- $\mathrm{C}_{4} \mathrm{H}_{3} \mathrm{~S}-, \mathrm{PhNH}-; \mathrm{R}=-\mathrm{Me},-\mathrm{CHMe}_{2},-\mathrm{CH}_{2} \mathrm{Ph} ; \mathrm{X}=-\mathrm{H},-\mathrm{NHCOPh}$ $\mathrm{Y}=-\left(\mathrm{CH}_{2}\right)_{2}^{-},-\left(\mathrm{CH}_{2}\right)_{3}^{-},-\left(\mathrm{CH}_{2}\right)_{4},-\left(\mathrm{CH}_{2}\right)_{5}^{-},-\mathrm{CH}_{2} \mathrm{CHMeCH}_{2}^{-},-\mathrm{CH}_{2} \mathrm{CH}(\mathrm{t}-\mathrm{Bu}) \mathrm{CH}_{2}-$

\section{Figure 1}

\section{Results and Discussion}

The hydrazonoyl halides 1a-k were prepared by a modified literature procedure, ${ }^{18-22}$ and the nitrilimines 2 were generated in situ from 1 by reaction with triethylamine $\left(\mathrm{Et}_{3} \mathrm{~N}\right)$. The nonisolable nitrilimines $\mathbf{2}$ reacted readily with 1,4-cyclohexanedione methylhydrazone $\mathbf{3}$ affording the corresponding 1,2,4,5,10,11,13,14-octaazadispiro[5.2.5.2] hexadeca-2,12-dienes 4a-k (Scheme 1), owing to the high nucleophilicity of the nitrogen atom carrying the methyl group. The formation of compounds 4a-k involving the nucleophilic addition of the methyl hydrazone $\mathbf{3}$ to the nitrilimines 2 to give the non-isolable acyclic intermediates 5, which ultimately undergo intramolecular cyclization to dispiroheterocyclic compounds 4a-k (Scheme 2).

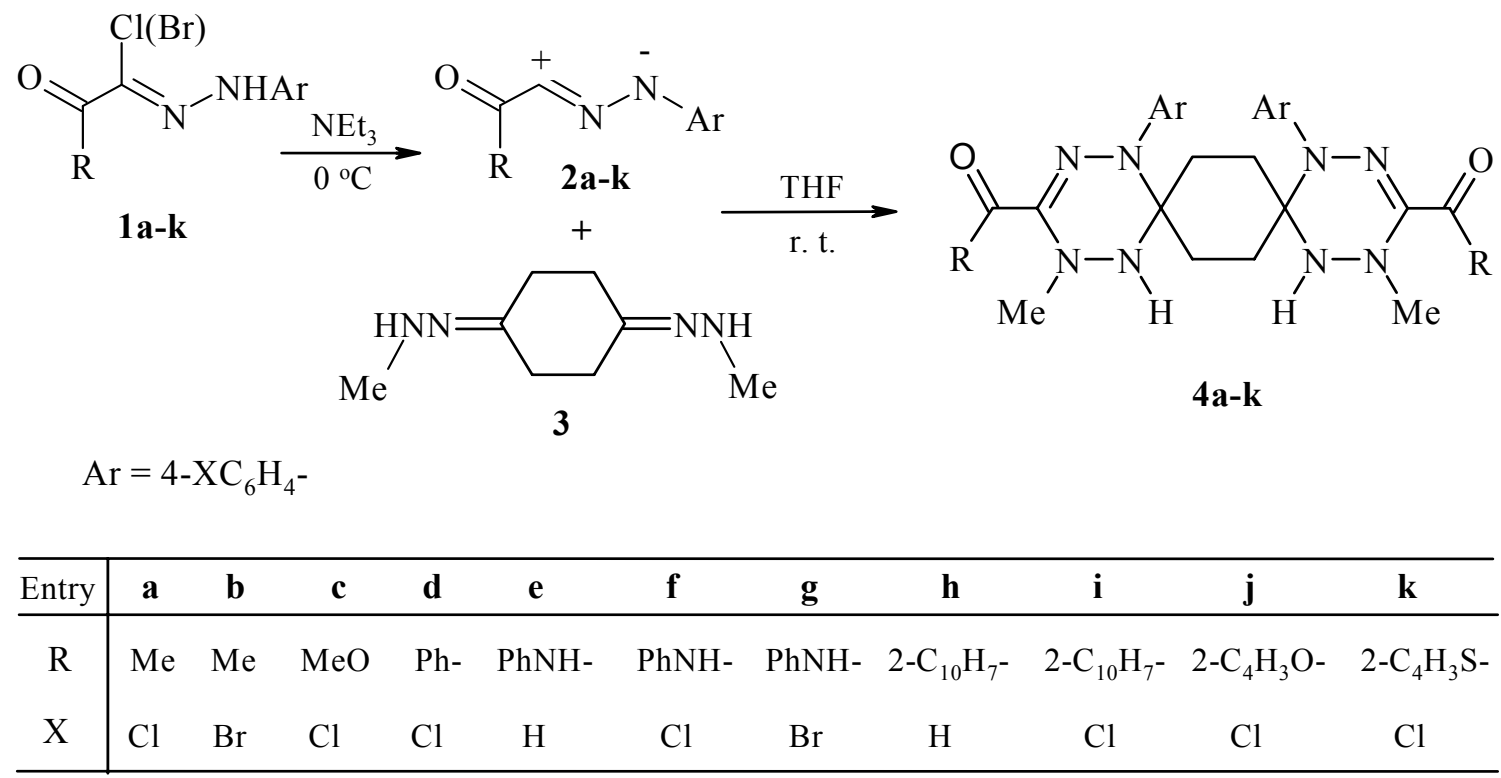

Scheme 1. Synthetic pathway for dispiro compounds 4a-k. 


\section{Spectral data analysis}

The structures of compounds $\mathbf{4 a - k}$ were established based on the basis of their elemental analysis and spectroscopical data. Physical properties, molecular ion peaks and elemental analysis for these triazinones are presented in the experimental section. The electron impact (EI) mass spectra displayed the correct molecular ions in accordance with the suggested structures. Their IR spectra showed absorption bands in the region 3280-3250 $\mathrm{cm}^{-1}$ and $1660-1640 \mathrm{~cm}^{-1}$ assignable to NH and carbonyl group, respectively. Their ${ }^{1} \mathrm{H}$ NMR spectra revealed, besides aromatic protons at 8.5-7.1 ppm and singlet signal at 3.2-3.0 ppm assigned to $\mathrm{NCH}_{3}$ protons and $\mathrm{D}_{2} \mathrm{O}$-exchangeble singlet signal in the region 5.7-5.6 ppm assignable to the ring $\mathrm{NH}$ proton. The detailed ${ }^{1} \mathrm{H}$ NMR data is shown in the experimental section. Their ${ }^{13} \mathrm{C}$ NMR spectra showed all the signals corresponding to the proposed structures, especially C-6 and C-9 (spiro carbons) were found to resonate at about 75$70 \mathrm{ppm}$. This is similar to reported values of spiro carbons flanked by two nitrogen atoms in sixmembered heterocycles, ${ }^{14,22}$ which provide strong evidence in support of the structures $\mathbf{4 a - k}$. The complete ${ }^{13} \mathrm{C}$ NMR data are presented in experimental section.

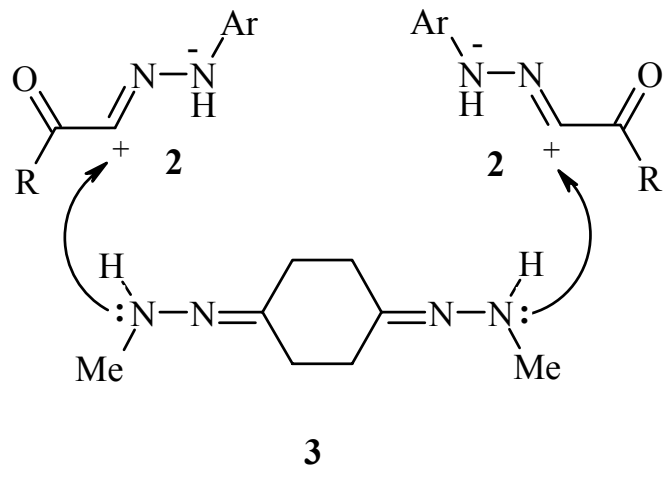

Nucleophilic addition
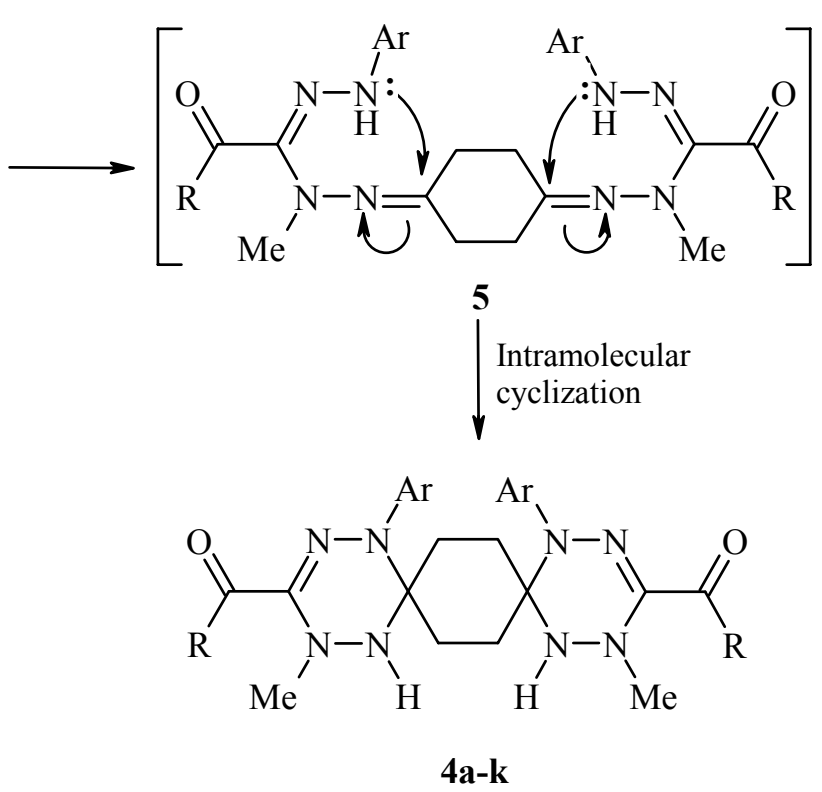

Scheme 2. The suggested reaction mechanism for compounds $4 \mathbf{a}-\mathbf{k}$.

\section{Biological activity}

Most of the newly synthesized compounds 4a-k were tested for their antibacterial and antifungal activity in vitro against bacterial strains such as Escherichia coli, Staphylococcus aureus, Klebsiella spp, Proteus spp, Pseudomonas and Aspergillus flavus, Candida albicans as fungi, employing the nutrient agar disc diffusion method ${ }^{23}$ at $(1-10 \mathrm{mg} / \mathrm{ml})$ in dimethyl formamide (DMF) by measuring the inhibition zone in $\mathrm{mm}$. It is interesting to note that these 
dispiroheterocycles 4a-k showed weak degree of activity against tested bacteria and fungi in comparison to DMF which was used as control. That may due to lack active substituted groups.

\section{Experimental Section}

General Procedures. All melting points were determined on an A. Krüss Melting Point Meter equipped with a digital thermometer and are uncorrected. The IR spectra were measured as potassium bromide pellets using a Satellite $3000 \mathrm{Mid}$ infrared spectrophotometer. The ${ }^{1} \mathrm{H}$ NMR and ${ }^{13} \mathrm{C}$ NMR spectra were recorded on a Bruker AM $300 \mathrm{MHz}$ spectrometer at room temperature in DMSO- $\mathrm{d}_{6}$ solution using tetramethylsilane (TMS) as internal reference. Chemical shifts were recorded as $\delta$ values in parts per millions (ppm) downfield from internal TMS. Electron impact (EI) mass spectra were run on Shimadzu GCMS-QP1000 EX spectrometer at 70 eV. Elemental analysis was performed at Cairo University, Egypt. The hydrazonoyl halides 1a$\mathbf{k}^{18-22}$ and 1,4-cyclohexanedione methylhydrazone $\mathbf{3}^{24}$ were prepared according to literature procedures. Tetrahydrofuran (THF) and triethylamine were purchased from Avocado Research Chemicals, England, and used without further purification.

\section{Reaction of nitrilimines 2 with 1,4-cyclohexanedione methylhydrazones 3}

Triethylamine $(0.05 \mathrm{~mol}, 7 \mathrm{ml})$ in tetrahydrofuran $(10 \mathrm{ml})$ was added dropwise to stirred mixture of a hydrazone $3(0.02 \mathrm{~mol})$ and the appropriate hydrazonoyl halides 1a-k $(0.01 \mathrm{~mol})$ in tetrahydrofuran $(70 \mathrm{ml})$ at $-5-0{ }^{\circ} \mathrm{C}$. The reaction temperature was allowed to rise slowly to ambient temperature and stirring was continued for 4-6 hours. The precipitated triethylammonium salt was filtered off and the solvent was then evaporated. The residue was washed with water $(100 \mathrm{ml})$ and in few cases the gummy products were triturated with ethanol $(10 \mathrm{ml})$. The crude solid product was collected and recrystallized from ethanol to give the desired compounds. The following compounds were synthesized using this method:

3,12-Diacetyl-1,14-di(4-chlorophenyl)-4,10-dimethyl-1,2,4,5,10,11,13,14-octaazadispiro[5.2.5.2] hexadeca-2,12-diene (4a). Yield 57\%, m.p. 139-141 ${ }^{\circ} \mathrm{C}$. IR (KBr): $\mathrm{cm}^{-1} 3285(\mathrm{NH}), 1682$ $(\mathrm{C}=\mathrm{O}), 1622(\mathrm{C}=\mathrm{N}) .{ }^{1} \mathrm{H}$ NMR $\left(\mathrm{DMSO}_{\mathrm{d}}\right)$ ): $\delta / \mathrm{ppm}: 8.52-7.25(\mathrm{~m}, 8 \mathrm{H}$, arom. protons), $5.74(\mathrm{~s}, 2 \mathrm{H}$, 2NH), $3.05\left(\mathrm{~s}, 6 \mathrm{H}, 2 \mathrm{CH}_{3}\right), 2.56\left(\mathrm{~s}, 6 \mathrm{H}, 2 \mathrm{CH}_{3}\right), 2.10-1.83\left(\mathrm{~m}, 8 \mathrm{H}, 4 \mathrm{CH}_{2}\right) .{ }^{13} \mathrm{C}$ NMR (DMSO-d 6 ): $\delta /$ ppm $187.10(\mathrm{C}=\mathrm{O}), 143.90-126.10\left(\mathrm{C}=\mathrm{N}\right.$, arom. carbons), 75.10 (spiro carbons), $43.45\left(\mathrm{NCH}_{3}\right)$, 28.07, $26.83\left(2 \mathrm{CH}_{2}\right)$. MS: $\mathrm{m} / \mathrm{z}=556 / 558\left(\mathrm{M}^{+}\right.$, Chlorine isotopes). Anal. for $\mathrm{C}_{26} \mathrm{H}_{30} \mathrm{Cl}_{2} \mathrm{~N}_{8} \mathrm{O}_{2}$ (557.49): Calcd. C, 56.02; H, 5.42; N, 20.10\%. Found: C, 56.20; H, 5.60; N, 19.90\%.

\section{3,12-Diacetyl-1,14-di(4-bromophenyl)-4,10-dimethyl-1,2,4,5,10,11,13,14-octaazadispiro-}

[5.2.5.2] hexadeca-2,12-diene (4b). Yield 55\%, m.p. 158-160 ${ }^{\circ} \mathrm{C}$. IR (KBr): $\mathrm{cm}^{-1} 3285(\mathrm{NH}), 1681$ $(\mathrm{C}=\mathrm{O}), 1620(\mathrm{C}=\mathrm{N}) .{ }^{1} \mathrm{H}$ NMR $\left(\mathrm{DMSO}_{\mathrm{d}}\right)$ ): $\delta / \mathrm{ppm}$ 8.52-7.25 (m, 8H, arom. protons), 5.74 (s, 2H, 2NH), $3.05\left(\mathrm{~s}, 6 \mathrm{H}, 2 \mathrm{CH}_{3}\right), 2.57\left(\mathrm{~s}, 6 \mathrm{H}, 2 \mathrm{CH}_{3}\right), 2.10-1.83\left(\mathrm{~m}, 8 \mathrm{H}, 4 \mathrm{CH}_{2}\right) .{ }^{13} \mathrm{C} \mathrm{NMR}\left(\mathrm{DMSO}-\mathrm{d}_{6}\right)$ :

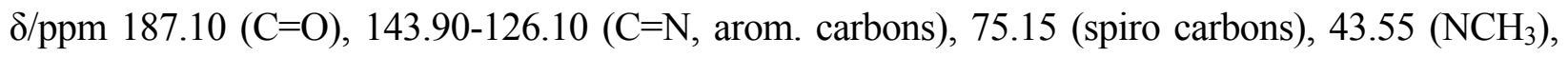


28.10, 26.86 $\left(2 \mathrm{CH}_{2}\right)$. MS: $\mathrm{m} / \mathrm{z}=646 / 648\left(\mathrm{M}^{+*}\right.$, Bromine isotopes). Anal. for $\mathrm{C}_{26} \mathrm{H}_{30} \mathrm{Br}_{2} \mathrm{~N}_{8} \mathrm{O}_{2}$ (646.39): Calcd. C, 48.31; H, 4.68; N, 17.34\%. Found: C, 48.80; H, 4.50; N, 17.20\%.

1,14-Di(4-chlorophenyl)-4,10-dimethyl-3,12-dimethoxycarbonyl-1,2,4,5,10,11,13,14-octaazadispiro[5.2.5.2] hexadeca-2,12-diene (4c). Yield 48\%, m.p. 137-139 ${ }^{\circ} \mathrm{C}$. IR (KBr): $\mathrm{cm}^{-1} 3280$ $(\mathrm{NH}), 1720(\mathrm{C}=\mathrm{O}), 1625(\mathrm{C}=\mathrm{N}) .{ }^{1} \mathrm{H}$ NMR $\left(\mathrm{DMSO}_{6} \mathrm{~d}_{6}\right): \delta / \mathrm{ppm}$ 8.52-7.25 (m, 8H, arom. protons), $5.74(\mathrm{~s}, 2 \mathrm{H}, 2 \mathrm{NH}), 3.76\left(\mathrm{~s}, 6 \mathrm{H}, 2 \mathrm{OCH}_{3}\right), 3.05\left(\mathrm{~s}, 6 \mathrm{H}, 2 \mathrm{CH}_{3}\right), 2.10-1.83\left(\mathrm{~m}, 8 \mathrm{H}, 4 \mathrm{CH}_{2}\right) ;{ }^{13} \mathrm{C} \mathrm{NMR}$ (DMSO-d $\left.\mathrm{d}_{6}\right): \delta /$ ppm $156.32(\mathrm{O}-\mathrm{C}=\mathrm{O}), 143.40-124.99(\mathrm{C}=\mathrm{N}$, arom. carbons), 74.92 (spiro carbons), $53.75\left(\mathrm{OCH}_{3}\right), 43.23\left(\mathrm{NCH}_{3}\right), 27.16,26.52\left(2 \mathrm{CH}_{2}\right) . \mathrm{MS}: \mathrm{m} / \mathrm{z}=588 / 590\left(\mathrm{M}^{+*}\right.$, Chlorine isotopes). Anal. for $\mathrm{C}_{26} \mathrm{H}_{30} \mathrm{Cl}_{2} \mathrm{~N}_{8} \mathrm{O}_{4}$ (589.49): Calcd. C, 52.98; H, 5.13; N, 19.01\%. Found: C, 52.70; H, 5.00; N, $18.90 \%$.

3,12-Dibenzoyl-1,14-di(4-chlorophenyl)-4,10-dimethyl-1,2,4,5,10,11,13,14-octaazadispiro[5.2.5.2]hexadeca-2,12-diene (4d). Yield 51\%, m.p. 192-194 ${ }^{\circ} \mathrm{C}$. IR (KBr): $\mathrm{cm}^{-1} 3265$ (NH), 1660 $(\mathrm{C}=\mathrm{O}), 1618(\mathrm{C}=\mathrm{N}) .{ }^{1} \mathrm{H}$ NMR (DMSO-d $)$ ) $\delta / \mathrm{ppm}$ 8.52-7.25 (m, 18H, arom. protons), 5.74 (s, 2H, 2NH), $3.05\left(\mathrm{~s}, 6 \mathrm{H}, 2 \mathrm{CH}_{3}\right), 2.10-1.83\left(\mathrm{~m}, 8 \mathrm{H}, 4 \mathrm{CH}_{2}\right) .{ }^{13} \mathrm{C} \mathrm{NMR}\left(\mathrm{DMSO}_{6} \mathrm{~d}_{6}\right): \delta / \mathrm{ppm} 187.10(\mathrm{C}=\mathrm{O})$, 143.90-126.10 $\left(\mathrm{C}=\mathrm{N}\right.$, arom. carbons), 71.45 (spiro carbons), $43.10\left(\mathrm{NCH}_{3}\right), 27.30,26.76\left(2 \mathrm{CH}_{2}\right)$. MS: $\mathrm{m} / \mathrm{z}=680 / 682\left(\mathrm{M}^{+}\right.$, Chlorine isotopes). Anal. for $\mathrm{C}_{36} \mathrm{H}_{34} \mathrm{Cl}_{2} \mathrm{~N}_{8} \mathrm{O}_{2}$ (681.63): Calcd. C, 63.44; $\mathrm{H}$, 5.03; N, 16.44\%. Found: C, 63.30; H, 4.90; N, 16.35\%.

4,10-Dimethyl-1,14-diphenyl-3,12-diphenylaminocarbonyl-1,2,4,5,10,11,13,14-octaazadispiro[5.2.5.2] hexadeca-2,12-diene (4e). Yield 53\%, m.p. 176-178 ${ }^{\circ} \mathrm{C}$. IR (KBr): cm ${ }^{-1} 3275,3250$ $(\mathrm{NH}), 1650(\mathrm{C}=\mathrm{O}), 1615(\mathrm{C}=\mathrm{N}) .{ }^{1} \mathrm{H}$ NMR $\left(\mathrm{DMSO}_{-} \mathrm{d}_{6}\right)$ : $\delta / \mathrm{ppm} 9.30(\mathrm{~s}, 2 \mathrm{H}, 2 \mathrm{PhNH}), 7.80-7.06(\mathrm{~m}$, $20 \mathrm{H}$, arom. protons), $5.68(\mathrm{~s}, 2 \mathrm{H}, 2 \mathrm{NH}), 3.06\left(\mathrm{~s}, 6 \mathrm{H}, 2 \mathrm{CH}_{3}\right), 2.13-1.63\left(\mathrm{~m}, 8 \mathrm{H}, 4 \mathrm{CH}_{2}\right) .{ }^{13} \mathrm{C} \mathrm{NMR}$ (DMSO-d $\left.\mathrm{d}_{6}\right): \delta / \mathrm{ppm} 158.60(\mathrm{C}=\mathrm{O}), 143.90-126.10(\mathrm{C}=\mathrm{N}$, arom. carbons), 70.55 (spiro carbons), $42.90\left(\mathrm{NCH}_{3}\right), 27.50,26.90\left(2 \mathrm{CH}_{2}\right)$. MS: $\mathrm{m} / \mathrm{z}=642\left(\mathrm{M}^{+\cdot}\right)$. Anal. for $\mathrm{C}_{36} \mathrm{H}_{38} \mathrm{~N}_{10} \mathrm{O}_{2}$ (642.77): Calcd. C, 67.27; H, 5.96; N, 21.79\%. Found: C, 67.00; H, 6.20; N, 21.80\%.

1,14-Di(4-chlorophenyl)-4,10-dimethyl-3,12-diphenylaminocarbonyl-1,2,4,5,10,11,13,14-octaazadispiro[5.2.5.2] hexadeca-2,12-diene (4f). Yield 50\%, m.p. 163-165 ${ }^{\circ} \mathrm{C}$. IR (KBr): $\mathrm{cm}^{-1} 3270$, $3255(\mathrm{NH}), 1655(\mathrm{C}=\mathrm{O}), 1612(\mathrm{C}=\mathrm{N}) .{ }^{1} \mathrm{H}$ NMR $\left(\mathrm{DMSO}_{-} \mathrm{d}_{6}\right): \delta / \mathrm{ppm} 9.10(\mathrm{~s}, 2 \mathrm{H}, 2 \mathrm{PhNH}), 7.82-7.10$ (m, 18H, arom. protons), $5.67(\mathrm{~s}, 2 \mathrm{H}, 2 \mathrm{NH}), 3.06\left(\mathrm{~s}, 6 \mathrm{H}, 2 \mathrm{CH}_{3}\right), 2.11-1.60\left(\mathrm{~m}, 8 \mathrm{H}, 4 \mathrm{CH}_{2}\right) .{ }^{13} \mathrm{C} \mathrm{NMR}$ (DMSO-d $\left.\mathrm{d}_{6}\right): \delta / \mathrm{ppm} 158.80(\mathrm{C}=\mathrm{O}), 143.90-126.10(\mathrm{C}=\mathrm{N}$, arom. carbons), 70.60 (spiro carbons), $42.90\left(\mathrm{NCH}_{3}\right), 27.70,26.96\left(2 \mathrm{CH}_{2}\right) . \mathrm{MS}: \mathrm{m} / \mathrm{z}=710 / 712\left(\mathrm{M}^{+\cdot}\right.$, Chlorine isotopes $)$. Anal. for $\mathrm{C}_{36} \mathrm{H}_{36} \mathrm{Cl}_{2} \mathrm{~N}_{10} \mathrm{O}_{2}$ (711.66): Calcd. C, 60.76; H, 5.10; N, 19.68\%. Found: C, 60.55; H, 4.85; N, $19.60 \%$.

1,14-Di(4-bromophenyl)-4,10-dimethyl-3,12-diphenylaminocarbonyl-1,2,4,5,10,11,13,14-octa-

azadispiro[5.2.5.2] hexadeca-2,12-diene (4g). Yield 51\%, m.p. 181-183 ${ }^{\circ} \mathrm{C}$. IR (KBr): $\mathrm{cm}^{-1} 3275$, $3252(\mathrm{NH}), 1651(\mathrm{C}=\mathrm{O}), 1610(\mathrm{C}=\mathrm{N}) .{ }^{1} \mathrm{H}$ NMR $\left(\mathrm{DMSO}-\mathrm{d}_{6}\right): \delta / \mathrm{ppm} 9.20(\mathrm{~s}, 2 \mathrm{H}, 2 \mathrm{PhNH}), 7.82-7.10$ (m, 18H, arom. protons), $5.65(\mathrm{~s}, 2 \mathrm{H}, 2 \mathrm{NH}), 3.05\left(\mathrm{~s}, 6 \mathrm{H}, 2 \mathrm{CH}_{3}\right), 2.12-1.62\left(\mathrm{~m}, 8 \mathrm{H}, 4 \mathrm{CH}_{2}\right) .{ }^{13} \mathrm{C} \mathrm{NMR}$ (DMSO-d $\left.\mathrm{d}_{6}\right): \delta / \mathrm{ppm} 158.80(\mathrm{C}=\mathrm{O}), 143.80-126.00(\mathrm{C}=\mathrm{N}$, arom. carbons), 70.62 (spiro carbons), $42.92\left(\mathrm{NCH}_{3}\right), 27.68,26.94\left(2 \mathrm{CH}_{2}\right) . \mathrm{MS}: \mathrm{m} / \mathrm{z}=800 / 802\left(\mathrm{M}^{+\cdot}\right.$, Bromine isotopes $)$. Anal. for $\mathrm{C}_{36} \mathrm{H}_{36} \mathrm{Br}_{2} \mathrm{~N}_{10} \mathrm{O}_{2}$ (800.56): Calcd. C, 54.01; H, 4.53; N, 17.50\%. Found: C, 53.95; H, 4.65; N, $17.60 \%$. 


\section{4,10-Dimethyl-3,12-di(2-naphthoyl)-1,14-diphenyl-1,2,4,5,10,11,13,14-octaazadispiro-}

[5.2.5.2] hexadeca-2,12-diene (4h). Yield 53\%, m.p. 188-190 ${ }^{\circ} \mathrm{C}$. IR (KBr): $\mathrm{cm}^{-1} 3265$ (NH), 1645 $(\mathrm{C}=\mathrm{O}), 1605(\mathrm{C}=\mathrm{N}) .{ }^{1} \mathrm{H}$ NMR $\left(\mathrm{DMSO}_{\mathrm{d}}\right)$ : $\delta / \mathrm{ppm}$ 8.50-7.22 (m, 24H, arom. protons), $5.72(\mathrm{~s}, 2 \mathrm{H}$, 2NH), $3.07\left(\mathrm{~s}, 6 \mathrm{H}, 2 \mathrm{CH}_{3}\right), 2.12-1.84\left(\mathrm{~m}, 8 \mathrm{H}, 4 \mathrm{CH}_{2}\right) .{ }^{13} \mathrm{C} \mathrm{NMR}\left(\mathrm{DMSO}_{6} \mathrm{~d}_{6}\right): \delta / \mathrm{ppm} 186.62(\mathrm{C}=\mathrm{O})$, 143.21-125.00 ( $\mathrm{C}=\mathrm{N}$, arom. carbons), 70.10 (spiro carbons), $43.33\left(\mathrm{NCH}_{3}\right), 27.35,26.78\left(2 \mathrm{CH}_{2}\right)$. MS: $\mathrm{m} / \mathrm{z}=712\left(\mathrm{M}^{+\cdot}\right)$. Anal. for $\mathrm{C}_{44} \mathrm{H}_{40} \mathrm{~N}_{8} \mathrm{O}_{2}$ (712.86): Calcd. C, 74.14; H, 5.66; N, 15.72\%. Found: C, 74.30; H, 5.75; N, 15.61\%.

1,14-Di(4-chlorophenyl)-4,10-dimethyl-3,12-di(2-naphthoyl)-1,2,4,5,10,11,13,14-octaazadispiro[5.2.5.2] hexadeca-2,12-diene (4i). Yield 50\%, m.p. $169-171{ }^{\circ} \mathrm{C}$. IR (KBr): $\mathrm{cm}^{-1} 3265(\mathrm{NH})$, $1645(\mathrm{C}=\mathrm{O}), 1605(\mathrm{C}=\mathrm{N}) .{ }^{1} \mathrm{H}$ NMR $\left(\mathrm{DMSO}-\mathrm{d}_{6}\right): \delta / \mathrm{ppm} 8.52-7.25(\mathrm{~m}, 22 \mathrm{H}$, arom. protons), $5.74(\mathrm{~s}$, $2 \mathrm{H}, 2 \mathrm{NH}), 3.05\left(\mathrm{~s}, 6 \mathrm{H}, 2 \mathrm{CH}_{3}\right), 2.10-1.83\left(\mathrm{~m}, 8 \mathrm{H}, 4 \mathrm{CH}_{2}\right) .{ }^{13} \mathrm{C} \mathrm{NMR}\left(\mathrm{DMSO}-\mathrm{d}_{6}\right): \delta / \mathrm{ppm} 186.66$ $(\mathrm{C}=\mathrm{O}), 143.40-125.00\left(\mathrm{C}=\mathrm{N}\right.$, arom. carbons), 70.06 (spiro carbons), $43.30\left(\mathrm{NCH}_{3}\right), 27.30,26.76$ $\left(2 \mathrm{CH}_{2}\right)$. MS: $\mathrm{m} / \mathrm{z}=780 / 782\left(\mathrm{M}^{+\cdot}\right.$, Chlorine isotopes). Anal. for $\mathrm{C}_{44} \mathrm{H}_{38} \mathrm{Cl}_{2} \mathrm{~N}_{8} \mathrm{O}_{2}$ (781.75): Calcd. C, 67.60; H, 4.90; N, 14.33\%. Found: C, 67.50; H, 4.75; N, 13.20\%.

\section{1,14-Di(4-chlorophenyl)-3,12-di(2-furoyl)-4,10-dimethyl-1,2,4,5,10,11,13,14-octaazadispiro-}

[5.2.5.2] hexadeca-2,12-diene (4j). Yield 49\%, m.p. 149-151 ${ }^{\circ} \mathrm{C}$. IR (KBr): cm $3270(\mathrm{NH}), 1665$ $(\mathrm{C}=\mathrm{O}), 1615(\mathrm{C}=\mathrm{N}) .{ }^{1} \mathrm{H}$ NMR (DMSO-d $): \delta / p p m ~ 7.82-7.11(\mathrm{~m}, 14 \mathrm{H}$, arom. protons), $5.64(\mathrm{~s}, 2 \mathrm{H}$, 2NH), $3.05\left(\mathrm{~s}, 6 \mathrm{H}, 2 \mathrm{CH}_{3}\right), 2.16-1.65\left(\mathrm{~m}, 8 \mathrm{H}, 4 \mathrm{CH}_{2}\right) ;{ }^{13} \mathrm{C} \mathrm{NMR}\left(\mathrm{DMSO}-\mathrm{d}_{6}\right): \delta / \mathrm{ppm} 174.60(\mathrm{C}=\mathrm{O})$, 143.50-127.77 $\left(\mathrm{C}=\mathrm{N}\right.$, arom. carbons), 71.22 (spiro carbons), $43.07\left(\mathrm{NCH}_{3}\right), 27.30,26.76\left(2 \mathrm{CH}_{2}\right)$. MS: $\mathrm{m} / \mathrm{z}=660 / 662\left(\mathrm{M}^{+\cdot}\right.$, Chlorine isotopes). Anal. for $\mathrm{C}_{32} \mathrm{H}_{30} \mathrm{Cl}_{2} \mathrm{~N}_{8} \mathrm{O}_{4}$ (661.55): Calcd. C, 58.10; $\mathrm{H}$, 4.57; N, 16.94\%. Found: C, 57.85; H, 4.40; N, 16.85\%.

1,14-Di(4-chlorophenyl)-4,10-dimethyl-3,12-di(2-thenoyl)-1,2,4,5,10,11,13,14-octaazadispiro[5.2.5.2] hexadeca-2,12-diene (4k). Yield 51\%, m.p. 180-182 ${ }^{\circ} \mathrm{C}$. IR (KBr): cm ${ }^{-1} 3275(\mathrm{NH})$, $1655(\mathrm{C}=\mathrm{O}), 1610(\mathrm{C}=\mathrm{N}) .{ }^{1} \mathrm{H}$ NMR $\left(\mathrm{DMSO}-\mathrm{d}_{6}\right): \delta / \mathrm{ppm} 7.89-7.09(\mathrm{~m}, 14 \mathrm{H}$, arom. protons), $5.65(\mathrm{~s}$, $2 \mathrm{H}, 2 \mathrm{NH}), 3.05\left(\mathrm{~s}, 6 \mathrm{H}, 2 \mathrm{CH}_{3}\right), 2.15-1.62\left(\mathrm{~m}, 8 \mathrm{H}, 4 \mathrm{CH}_{2}\right) ;{ }^{13} \mathrm{C} \mathrm{NMR}\left(\mathrm{DMSO}_{6} \mathrm{~d}_{6}\right): \delta / \mathrm{ppm} 177.37$ $(\mathrm{C}=\mathrm{O}), 143.70-127.38\left(\mathrm{C}=\mathrm{N}\right.$, arom. carbons), 71.17 (spiro carbons), $43.11\left(\mathrm{NCH}_{3}\right), 27.10,26.20$ $\left(2 \mathrm{CH}_{2}\right) . \mathrm{MS}: \mathrm{m} / \mathrm{z}=692 / 694\left(\mathrm{M}^{+}\right.$, Chlorine isotopes). Anal. for $\mathrm{C}_{32} \mathrm{H}_{30} \mathrm{Cl}_{2} \mathrm{~N}_{8} \mathrm{O}_{2} \mathrm{~S}_{2}$ (693.68): Calcd. C, 55.41; H, 4.36; N, 16.15\%. Found: C, 55.20; H, 4.20; N, 16.00\%.

\section{Acknowledgements}

The author is thankful to the Union of Arab Universities (UAU), Supporting Box of Palestinian Universities, Amman, Jordan, for financial support. Also thanks to Dr. A. S. Abu- Samaha, Biology Department, Alaqsa University of Gaza, for providing antimicrobial testing facility for the synthesized compounds. 


\section{References and Footnotes}

1. Mashelkar, U. C.; Rane, D. M. Synthesis of Some Isatin Based Novel Spiroheterocycles and Their Biological Activity Studies. Ind. J. Chem. 2005, 44B, 1937-1939.

2. Kisel, V. M.; Kostyrko, E. O.; Kovtunenko, V. A. Synthesis and Biological Properties of Isoquinolines Spirofused with Carbocycles and Heterocycles at Position 4. Chem. Heterocycl. Comp. 2004, 38, 1295-1318.

3. Güzel, Ö.; Ilhan, E.; Salman, A. Synthesis and Antimycobacterial Activity of New 2-HydroxyN-(3-oxo-1-thia-4-azaspiro[4.4]non-4-yl)/(3-oxo-1-thia-4-azaspiro[4.5]dec-4-yl)-2,2diphenylacetamide Derivatives. Monatsh. Chem. 2006, 137, 795-801.

4. Alahmadi, A.A.; El-Zohry, M. F. Synthesis of Some Spiroheterocyclic Pyrylium-Salts Related to 1-oxa-4-thiaspiro[4.4]nonan-2-one and/or [4.5]decan-2-one as antimicrobial agents. $J$. Chem. Technol. Biotechnol. 1995, 62, 366-372.

5. Chen, M-H.; Pollard, P. P.; Patchett, A. A.; Cheng, K.; Wei, L.; Chan, W.; Butler, B.; Jacks, T. M.; Smith, R. G. Synthesis and Biological Activities of Spiroheterocyclic Growth Hormone Secretagogues. Bioorg. Med. Chem. Lett. 1999, 9, 1261-1266.

6. Joshi, N. S.; Karale, B. K.; Gill, C. H. Synthesis of Some Thiadiazoles, Selenadiazoles and Spiroheterocyclic Compounds from Their 2,2-Dimethylbenzopyrane Precursors. Chem. Heterocycl. Comp. 2006, 42, 681-685.

7. H-Zahmani, H.; Viala, J.; Hacini, S.; Rodriguez, J. Synthesis of Functionalized Spiroheterocycles by Sequential Multicomponent Reaction/Metal-Catalyzed Carbo-cyclization from Simple $\beta$-Ketoesters and Amides. Synlett. 2007, 1037-1042.

8. Kuroyan, R. A. Synthesis of Spiro[Piperidine-4, $\mathrm{C}_{\mathrm{n}}{ }^{\prime}$-hetero(carbo)cycles]. Russ. Chem. Rev. 1991, 60, 1368-1383; Awad, I. M.; Hafez, A. A.; El-Zohry, M. F. Spiroheterocyclic System. IV: Novel Azo Dyes Sulfa Drugs Spiroheterocyclic Naphthenes. J. Chem. Technol. Biotechnol. 1992, 55, 293-307.

9. Mckibben, R. K. Spiroheterocyclic Compounds, Process, for Their Preparation and Pharmaceutical Compositions Containing Them. Eur. Pat. EP 0743312, 2007.

10. Lukyanov, B. S.; Lukyanov, M. B. Spiropyrans: Synthesis, Properties and Application. Chem. Heterocycl. Comp. 2005, 41, 281-311.

11. Arutyunyan, G. L.; Chachoyan, A. A. Agadzhanyan, Ts. E.; Garibdzhanyan, B. T. Synthesis and Antitumor Properties of Some Spiroheterocyclic 1,3-Diazaadamantanes. Pharm. Chem. J. 1996, 30, 739-741.

12. Güzel, Ö.; Terzioğlu, N.; Çapan, G.; Salman, A. Synthesis and biological evaluation of new5-methyl- $N$-(3-oxo-1-thia-4-azaspiro[4.5]-dec-4-yl)-3-phenyl-1 $H$-indole-2-carbox-amide derivatives. Arkivoc 2006, (xii), 98-110.

13. Ferwanah, A. R. S.; Awadallah, A. M. Reaction of Nitrilimines and Nitrile Oxides with Hydrazines, Hydrazones and Oximes. Molecules 2005, 10, 492-507. 
14. Dalloul, H. M.; Boyle, P. H. Heterocyclic Synthesis Using Nitrilimines: Part 3. Synthesis of Substituted 1,2,4,5-Tetrazines and 1,2,4,5-tetraaza-2-pentenes. Heterocycl. Commun. 2003, 9, 507-514.

15. Dalloul, H. M. Heterocyclic Synthesis Using Nitrilimines: Part 4. Synthesis of 3-Substituted 1-Aryl-1,2,4-triazaspiroalk-2-nes. Chem. Heterocycl. Comp. 2004, 40, 1402-1407.

16. Dalloul, H. M.; Boyle, P. H. Heterocyclic Synthesis Using Nitrilimines: Part 5. Synthesis of Some Novel Spiro Heterocycles. Turk. J. Chem. 2006, 30, 119-124.

17. Dalloul, H. M.; Boyle, P. H. Heterocyclic Synthesis Using Nitrilimines: Part 7. Synthesis of Some New 3-Substituted 1-Aryl-1,2,4,8-tetraazaspiro[4.5]dec-2enes. Heterocycl. Commun. 2007, 13, 155-160.

18. El-Abadelah, M. M.; Hussein, A. Q.; Thaher, B. A. Heterocycles from Nitrile Imines. Part IV. Chiral 4,5-Dihydro-1,2,4-triazin-ones. Heterocycles 1991, 32, 1879-1895; Shawali, A. S.; Abdelhamid, A. O. Reaction of Dimethylphenacylsulphonium Bromide with N-Nitrosoacetarylamides and Reactions of the Products with Nucleophiles. Bull. Chem. Soc. Jap. 1976, 49, 321324.

19. Hassaneen, H. M.; Shawali, A. S.; Elwan, N. M.; Abounada, N. M. Reaction of 1-(2Naphthoyl)methyl-2-dimethylsulfonium Bromide With N-Nitroso-N-aryl-acetamides and Reactions of the Products With Some Nucleophiles. Sulfur Lett. 1992, 13, 273-285; Hassaneen, H. M.; Shawali, A. S.; Elwan, N. M.; Abounada, N. M. Synthesis of Pyrazolo[3,4-d]pyridazine, Pyrazolo[3,4-d]pyrimidine and Imidazolo[1,2-a]pyridine Derivatives using Hydrazonyl Bromides, Org. Prep. Proced. Int. 1992, 24, 171-175.

20. Frohberg, P.; Drutkowski, G.; Wagner, C. Synthesis and Structural Assignment of Oxanilo-Narylhydrazonoyl Chlorides. Eur. J. Org. Chem. 2002, 1654-1663.

21. Shawali, A. S.; Hassaneen, H. M.; Shetta, A.; Osman, A.; Abdel-Galil, F. Heterocycles 1982, 19, 57-62; Farag, A. M.; Algharib, M. S. Org. Prep. Proced. Int. 1988, 20, 521-526.

22. El-Abadelah, M. M.; Hussein, A. Q.; Kamal, M. R.; Al-Adhami, K. H. Heterocycles from Nitrile Imines. Part I. 1,2,3,4-Tetrahydro-1,2,4,5-tetrazines. Heterocycles 1988, 27, 917-924; Awadallah, A. M.; Ferwanah, A. S.; Elsawi, E. A.; Dalloul, H. M. Reaction of C-Aroyl-Naryl Nitrilimines with Selected Aliphatic Keto-Hydrazones and Keto-Methyl-hydrazones, Asian J. Chem. 2002, 14, 1225-1229.

23. Collins, C. H. Microbiological Methods, Butterworths, London, 1967, p. 364.

24. Wiley, R. H.; Irick, G. Methyl and Dimethylhydrazones. J. Org. Chem. 1959, 24, 1925-1928; Todd, D. The Wolff-Kishner Reduction. 1. The Preparation and Properties of N-Alkyl- and N,N-Dialkylhydrazones. J. Am. Chem. Soc. 1949, 71, 1353-1355. 